\title{
Intranasal, live, attenuated influenza virus vaccine reduced severe febrile illness in healthy adults
}

Nichol KL, Mendelman PM, Mallon KP,et al, for the Live Attenuated Influenza Virus Vaccine in Healthy Adults Trial Group. Effectiveness of live, attenuated intranasal influenza virus vaccine in healthy, working adults: a randomized controlled trial.JAMA 1999 Jul 14;282:137-44.

QUESTION: Is an intranasal, trivalent, live, attenuated influenza virus (LAIV) vaccine effective and safe for reducing febrile illness in healthy, working adults?

\section{Design}

Randomised (allocation not concealed*), blinded (participants and study personnel),* placebo controlled trial with follow up during site specific 7 week peak outbreak periods.

Setting

13 sites in the continental United States.

\section{Participants}

4561 people (mean age 38 y, 55\% women) who were 18 to 64 years of age, worked $\geq 30 \mathrm{~h} /$ wk outside of the home, and had health insurance. Exclusion criteria included hypersensitivity to eggs, indication for or previous receipt of the inactivated vaccine, and pregnancy or unprotected risk for pregnancy in the previous 3 months. Follow up was $93 \%$.

\section{Intervention}

From September 18 to November 15, 1997, 3041 participants received the LAIV vaccine (A/Shenzhen/227/95 [HINI], A/Wuhan/359/95 [H3N2], and B/Harbin/7/ 94-like) administered in a single dose nasal spray. 1520 participants received the placebo vaccine.

\section{Main outcome measures}

3 illness categories were defined: febrile illness ( $\geq 2$ consecutive d of symptoms, with fever and $\geq 2$ symptoms each for $\geq 1 \mathrm{~d}$ ); severe febrile illness ( $\geq 3$ consecutive $\mathrm{d}$ of symptoms, with $\geq 1 \mathrm{~d}$ of fever and $\geq 2$ symptoms for $\geq 3 \mathrm{~d}$ ); and febrile upper respiratory tract infection (URTI) ( $\geq 2$ consecutive $d$ of URTI symptoms, fever for $\geq 1 \mathrm{~d}$, and 2 symptoms for $\geq 1 \mathrm{~d}$ ). Outcomes for each illness category included number of illness episodes, days of illness, days of missed work, and days with $\geq 1$ health care provider visit.

For correspondence: Dr K L Nichol,

Medicine Service (111),

Veterans Affairs

Medical Center, 1

Veterans Drive,

Minneapolis, $M N$

55417, USA. Fax

$+16127275659$

\section{Main results}

The groups did not differ for febrile illness episodes $(\mathrm{p}=0.10)$, but the vaccine group had lower rates of

Severe febrile illness outcomes in healthy, working adults receiving influenza vaccine v placebo

\begin{tabular}{lccc}
\multirow{2}{*}{$\begin{array}{l}\text { Outcomes during } 7 \text { wk peak } \\
\text { outbreaks }\end{array}$} & \multicolumn{2}{l}{ Rate/1000 persons } & \\
\cline { 2 - 3 } & Vaccine & Placebo & Rate reduction (95\% CI) \\
Number of illness episodes & 111 & 137 & $18.8 \%$ (7.4 to 28.8$)$ \\
\hline Days of illness & 1021 & 1404 & $27.3 \%$ (16.7 to 36.5$)$ \\
\hline Days of missed work & 155 & 188 & $17.9 \%(4.3$ to 29.5$)$ \\
\hline Days with $\geq 1$ health care visit & 37.6 & 50.1 & $24.8 \%$ (11.6 to 36.1$)$ \\
\hline
\end{tabular}

severe febrile illness episodes and related outcomes (table) and lower rates of febrile URTI episodes $(23.6 \%$ reduction in rates/1000, 95\% CI 12.7 to 33.2 ) and related outcomes. No serious adverse effects occurred in either group.

\section{Conclusion}

Intranasal, trivalent, live, attenuated influenza virus vaccine reduced severe febrile illness and outcomes related to febrile upper respiratory tract illness in healthy, working adults.

*See glossary.

\section{COMMENTARY}

The limitations of the current inactivated influenza vaccine include need for an annual injection, poor induction of mucosal and cellular immune responses, and variable effectiveness, especially in such high risk groups as the elderly.

After intranasal administration, LAIV replicates, producing a mild sore throat or coryza and inducing serum IgG and mucosal IgA anti-influenza antibodies. Person to person spread of LAIV does not seem to occur, and there seems to be no risk for reversion to a wild-type phenotype. ${ }^{1}$

Nichol and colleagues showed that intranasal, trivalent LAIV was safe and effective in healthy adults aged 18 to 64 years. The primary end point was febrile illnesses during influenza outbreak periods, which tends to underestimate the vaccine's true efficacy. For example, in children, the LAIV vaccine was 93\% effective against culture confirmed influenza but only $25 \%$ to $30 \%$ effective in reducing febrile episodes. $^{2}$

Unlike inactivated vaccine, which induces type specific IgG antibodies, the broader protection of the LAIV vaccine might result from induction of cross-reactive mucosal IgA antibodies. ${ }^{1}$ A study that directly compared a bivalent LAIV with inactivated vaccines, however, did not show any advantage for LAIV vaccine when the circulating influenza virus was a poor antigenic match. ${ }^{3}$ No data are published on its use in immunocompromised patients; in older people, it will probably be used as a supplement to the inactivated vaccine.

The LAIV vaccine will probably be licensed within the next year. In addition to its potential immunological advantage, it might also have higher patient acceptability: it does not require a needle, and about two thirds of the recipients readily self administered LAIV vaccine. Consumers can expect to pay for this convenience.

Bradley S Bender, MD Veterans Affairs Medical Center Gainesville, Florida, USA

1 Subbarao K. Adv Virus Res 1999;54:349-73.

2 Belshe RB, Mendelman PM, Treanor J, et al. N Engl J Med 1998;338:1405-12.

3 Edwards KM, Dupont WD, Westrich MK, et al. J Infect Dis 1994;169:625-33.

4 Treanor JJ, Mattison HR, Dumyati G, et al. Ann Intern Med 1992;117:625-33 